\title{
Risk management support through India Remote Sensing Satellites
}

\author{
N Aparna ${ }^{\text {a, }}$, A V Ramani ${ }^{\text {b }} \&$ R Nagaraja ${ }^{c}$ \\ a Scientist Engineer "SG”, Head Mission Interface and User Services NRSC-NDC - aparna_n@ nrsc.gov.in \\ ${ }^{\mathrm{b}}$ Scientist Engineer "SF" MI\&US NRSC-NDC -ramani_av@nrsc.gov.in \\ ${ }^{\mathrm{c}}$ Group Director NDC-NRSC :nagaraja_r@nrsc.gov.in
}

Commission VI, WG VI/4

KEY WORDS: Payload planning, Emergency planning, Operational Remote Sensing Systems, GIS

\begin{abstract}
Remote Sensing along with Geographical Information System (GIS) has been proven as a very important tools for the monitoring of the Earth resources and the detection of its temporal variations. A variety of operational National applications in the fields of Crop yield estimation, flood monitoring, forest fire detection, landslide and land cover variations were shown in the last 25 years using the Remote Sensing data. The technology has proven very useful for risk management like by mapping of flood inundated areas identifying of escape routes and for identifying the locations of temporary housing or a-posteriori evaluation of damaged areas etc. The demand and need for Remote Sensing satellite data for such applications has increased tremendously. This can be attributed to the technology adaptation and also the happening of disasters due to the global climate changes or the urbanization. However, the real-time utilization of remote sensing data for emergency situations is still a difficult task because of the lack of a dedicated system (constellation) of satellites providing a day-to-day revisit of any area on the globe. The need of the day is to provide satellite data with the shortest delay. Tasking the satellite to product dissemination to the user is to be done in few hours. Indian Remote Sensing satellites with a range of resolutions from $1 \mathrm{~km}$ to $1 \mathrm{~m}$ has been supporting disasters both National \& International. In this paper, an attempt has been made to describe the expected performance and limitations of the Indian Remote Sensing Satellites available for risk management applications, as well as an analysis of future systems Cartosat-2D, 2E ,Resourcesat-2R \&RISAT-1A. This paper also attempts to describe the criteria of satellite selection for programming for the purpose of risk management with a special emphasis on planning RISAT-1(SAR sensor).
\end{abstract}

\section{INTRODUCTION}

ISRO has launched several satellites with multi sensors. Each sensor has a capability to provide the information of the surface of earth spatially and spectrally. These are unique information about the properties of the earth surface the land topography and the shallow waters. The satellites systems considered are optical and Radar. The Optical satellite systems considered here are , Resourcesat-2 , Oceansat-2 , Cartosat-2 \& Cartosat-1. The Radar satellite considered is RISAT-1. Earth observational optical systems are passive sensors, operating in the visible spectrum $(0.4 \mu-0.7 \mu)$ near infrared $(0.8 \mu$ to $0.9 \mu)$ and $(1.5 \mu$ $1.8 \mu)$ medium infrared. These measurements of easily interpretable but have the impact of the atmospheric conditions. Moreover whenever Cyclones, floods, forest fires, volcano's occur they are always accompanied with either clouds or thick smoke. This reduces the utilization of optical sensors operationally for disasters

RADAR sensors are active instruments they are independent of the sun illumination allowing both ascending and descending orbits for image acquisitions. High sensitivity to roughness and humidity also allows to retrieve different physical parameters compared to the optical sensors. The fact that they are "all weather" capable makes Radar sensors operational in emergency context.
For the context of emergency the revisit time and appropriate resolutions are very important parameters to be considered.

For example Oceansat- 2 which has a receptivity of 2 days cover the area every alternate day and gives a synoptic view of the area in 8 narrow spectral bands. This can be used for monitoring at broad level. In case of AWIFS we can look at the same area on every fifth day, with a resolution of $56 \mathrm{~m}$ and 12 bit radiometry. This helps in the flood inundations, effected area mapping and also monitoring at regular intervals. RISAT-1 can view the area every $2 / 3$ days. These satellites if planned and data is made available to users in timely manner, gives lot of information to the decision makers to manage and allocate the resources.

\subsection{Current Indian Remote Sensing satellites (IRS) constellation of satellites:}

The IRS optical and microwave satellites can be operated in multimode's.

Resourcesat-2 has three sensors allowing imaging at a resolution of $5.8 \mathrm{~m}$ to $56 \mathrm{~m}$, Cartosat- 1 can be operated in 2 modes, either stereo or wide. The Wide mode gives the advantage of covering larger area $\sim 56 \mathrm{~km}$ with $2.5 \mathrm{~m}$ resolution. Cartosat- 2 can be operated in Spot mode / strip mode or paint brush mode. The paint brush mode gives the maximum area coverage of $27 * 27 \mathrm{sq} \mathrm{km}$. RISAT-1 can be operated in four modes, FRS -1 FRS-2, CRS and MRS. The resolution ranges 
from $3 \mathrm{~m}$ to $50 \mathrm{~m}$. On demand the satellite can be operated to get high resolution data of $1 \mathrm{~m}$ for $10^{*} 100 \mathrm{sq} \mathrm{km}$. Following table- 1 gives the details of the satellites revisit capabilities available for Risk Management.

Table-1 IRS satellites available for risk management

\begin{tabular}{|l|l|l|l|l|}
\hline $\begin{array}{l}\text { S } \\
\text { NO }\end{array}$ & Satellite & Mode & $\begin{array}{l}\text { Revisit } \\
\text { in days }\end{array}$ & $\begin{array}{l}\text { Node } \\
\text { capability }\end{array}$ \\
\hline 1 & Resourcesat-2 & $\begin{array}{l}\text { a.LISS-1V } \\
\text { b.LISS-3 } \\
\text { c.AWiFS }\end{array}$ & $\begin{array}{l}\text { a.5 } \\
\text { b.24 } \\
\text { c.5 }\end{array}$ & $\begin{array}{l}\text { Only } \\
\text { Descending }\end{array}$ \\
\hline 2 & Cratosat-1 & PAN & 5 & $\begin{array}{l}\text { Only } \\
\text { Descending }\end{array}$ \\
\hline 3 & Cartosat-2 & PAN & $4 / 5$ & $\begin{array}{l}\text { Only } \\
\text { Descending }\end{array}$ \\
\hline 4 & RISAT-2 & $\begin{array}{l}\text { SAR X } \\
\text { Band }\end{array}$ & 2 & $\begin{array}{l}\text { Both } \\
\text { Descending } \\
\& \\
\text { Ascending }\end{array}$ \\
\hline 5 & RISAT-1 & $\begin{array}{l}\text { SAR C } \\
\text { Band }\end{array}$ & $2 / 3$ & $\begin{array}{l}\text { Both } \\
\text { Descending } \\
\& \\
\text { Ascending }\end{array}$ \\
\hline
\end{tabular}

The acquisitions \& planning for the purpose of damage assessment is very critical as the matching between archives and new acquisitions plays an essential role. We have also presented few case studies to show the capabilities of the existing operational satellites and the time lines achieved. A study has been made as to how frequently a given area can be covered with the existing IRS satellite constellations. The adequacy of the satellites is also worked out as the risk management needs continuous monitoring of disaster areas which then clashes with the National projects needs. The analysis show that it will be only with the launch of a dedicated constellation of satellites which allow simultaneous optical and radar acquisition with high repeat frequencies will help in operationalising the services for risk monitoring.

\subsection{Acquisition planning strategy for risk management}

In an operational scenario all the satellites have a short term plan and a long term plan. The long term plan is at weekly level and the short term plan is to accommodate any change or urgent request. The long term plan also takes care of the advance requests.

Following are the modes of planning the requests

- Long term planning

- Short term planning

- $\quad$ Day wise planning

The long term planning gathers all the user requests and calculates the the time left for some more operations and freezes the areas of collections. This helps us in building up archives.( Figure -1)

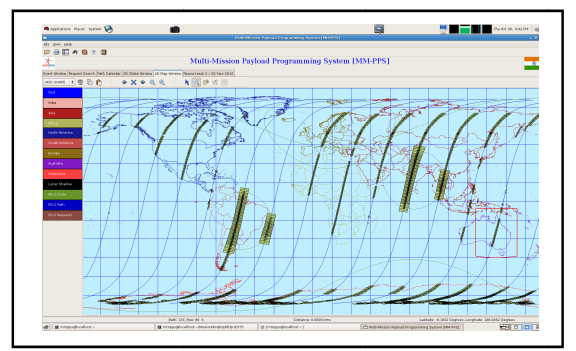

Figure:-1 : Consolidation of request
During emergencies all the long term plan and the short term plan will be overruled and the new request needs to be introduced. The request can be triggered at any time, if the schedules are not released the emergency will be included and the schedules will be generated. The new request inclusion is from an authorized login as shown in the flow chart-1.

\section{Flow chart-1 Showing the flow of the emergency request.}

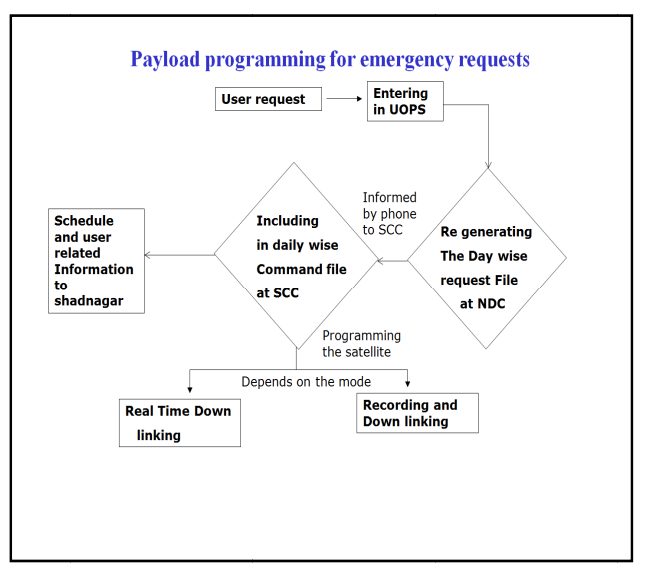

Three cases arise for an emergency request.

1. The schedules of next day are not yet released.

2. The schedules have been released but not uplinked to the satellite.

3. The commands have been uplinked.

Time Lines followed are:

- 1000 IST P/L Schedule Planning (PPS) for Next day.

- 1300 IST NDC Sends P/L Schedule File to SCC

- 1500 IST Commands are Generated at ISTRAC

- 1800 - 2330 IST Commands will be Uplinked to S/C .

- 0530 IST Onwards P/L Session Commands Execution Starts

CASE-1: If Emergency Exist before 1730 IST on Working Days, then the Emergency Request will be Ingested by payload planning team at NDC along with Regular Request and Send to SCC. If already Commands are Generated at ISTRAC \& not Uplinked Regenerate the Commands with new I/P from NDC.

CASE-2: If Emergency Exist After 1730 IST, and the Commands are not Uplinked to S/C. Though User Order Processing System ( UOPS) we ingest Emergency. PPS Generates Opportunities/proposal to UOPS online , enabling online proposals for the planners. After Confirmation PPS generates a separate file having Emergency Request \& the Time/Orbit Number from where the Emergency has been Included.

CSG - ISTRAC re-generates the Commands using New Emergency I/P + Daily Request File which was send earlier. Upload the SCH File to PPS for Automatic Pass Schedule File Generation

CASE-3: If Emergency Exist after Commands Uplinked to S/C Execution Started \& Few Sessions are already Executed.

After Confirmation PPS generates a separate file having Emergency Request \& the Time/Orbit Number from where the Emergency has been Included. 
CSG - ISTRAC regenerates partial (from where Emergency has been Included) Commands using New Emergency I/P + Daily Request File which was send earlier. Upload the SCH File to PPS for Automatic Pass Schedule File Generation.

Check against TTC schedule file, weather there are sufficient TTC Orbits for Commands unlinking ( To be Coordinated with S/C Controllers ). If there is no sufficient TTC Orbits for uplinking then Extra TTC Passes may be Taken by the Controller if Possible.

Therefore the possibility of best service for an emergency depends upon the above mentioned cases. Availability of orbits for TTC commanding is very essential.

Following are the steps whenever and emergency is triggered

$>$ Weather the area is within the Indian cone or outside

Checking the feasibility of acquisition for coming few days from on all available constellation of satellites

$>$ Coordination with Disaster Monitoring group/International charter to understand the requirement of Resolution ,frequency and time linel

$>$ Pre-event preparatory -data acquisition in the probable effected area

$>$ Decision on preferential dump orbits.

$>$ Decision on the additional area to be collected in and around the affected areas.

$>$ Initiating automatic processing trigger as soon as the data is down linked.

\subsection{Analysis of number of opportunities for a given area of} interest.

To arrive at the number of opportunities with the existing constellation of IRS satellites, a point of interest for collection is chosen and analysis is made. For the case study Resourcesat2, RISAT-1,CARTOSAT-2 and CARTOSAT-1 are considered. As a test case let us take the point (latitude 22.73 and longitude 85.87).

Let us analyze this with respect to the RISAT-1 satellite. RISAT-1 satellite can be operated both in Descending as well as Ascending node with Right or Left look. This gives permutation of $2 * 2=4$ ways of acquiring data as shown in the Figure- 2 . Figure- 3 shows the orbits of RISAT- 1 on a given day.

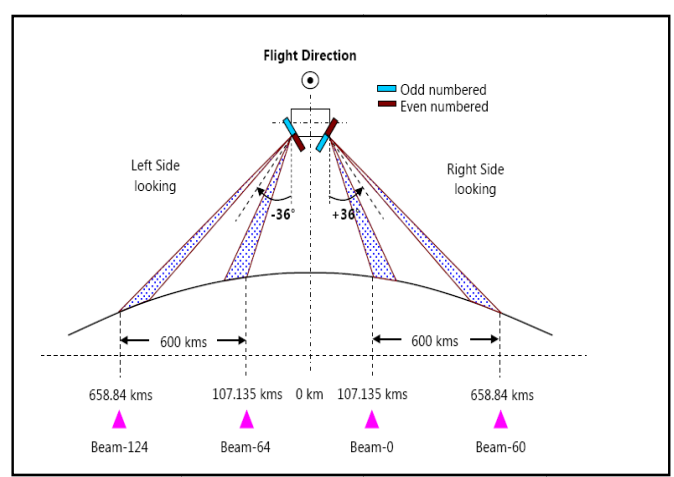

Figure :-2 RISAT-1 Left \& Right Looking

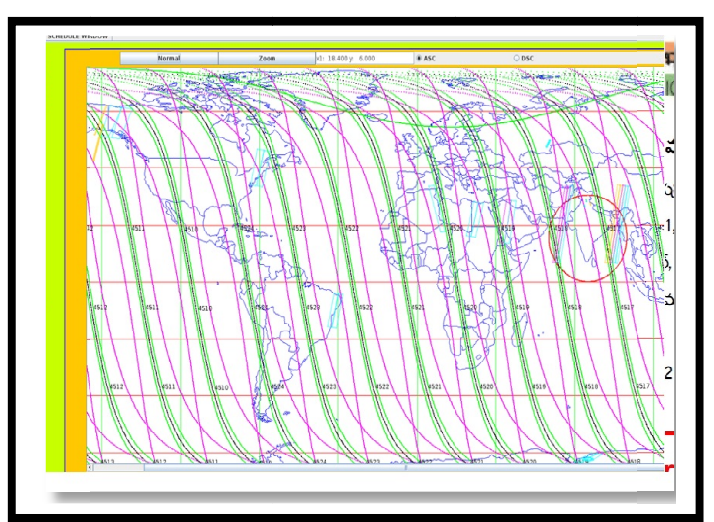

Figure :-3 RISAT-1 orbital pattern

With this the following are the feasibilities on given point of interest

\begin{tabular}{|l|l|}
\hline Satellite & Dates \\
\hline RISAT-1 & $16^{\text {th }}$ Nov \\
\hline & $17^{\text {th }}$ Nov \\
\hline & $20^{\text {th }}$ Nov \\
\hline & $21^{\text {st }}$ Nov \\
\hline & $22^{\text {nd }}$ Nov \\
\hline & $24^{\text {th }}$ Nov \\
\hline & $25^{\text {th }}$ Nov \\
\hline
\end{tabular}

A typical case study of a point with RISAT-1 for a period of $15^{\text {th }}$ Nov to 25th Nov 2014 shows that the opportunities are continuous from $16^{\text {th }}$ to $17^{\text {th }}$ and then from $20^{\text {th }}$ to $22^{\text {nd }}$ and again on $24^{\text {th }}$ and 25 th using both Ascending and Descending nodes. On $18^{\text {th }}$ and $19^{\text {th }}$ no possibility again on $23^{\text {rd }}$ no possibility.

The opportunities with Resourcesat- 2 are on $18^{\text {th }}$ Nov and $23^{\text {rd }}$ Nov.

Similarly if we generate the opportunities of Cartosat-1 \& Cartosat-2. It is observed that Cartosat-1 \& Cartosat-2 have the opportunities of collections on $18^{\text {th }}$ Nov 2014.As these satellites are not phased, in certain cases Cartosat-1 \& Catosat-2 have the collection opportunities on the same day. This sometime becomes a disadvantage and reduces the total number of opportunities. Therefore when coupled with the optical satellites the opportunity of collections are on a daily basis but not less than a day.

\subsection{Current status}

Remote Sensing satellites support in mapping the after effects of floods, hurricanes, earthquakes volcano eruptions, oil spill etc and also helps in prediction of Drought affected areas provide information on water contamination etc. Most of these have the problem of cloud when it comes to the optical sensors therefore the analysis of such kind of risk management / disaster management is more and more depending upon the microwave satellites.

Most of the risk management need continuous monitoring of a given area for assessment and relief activities. The problem needs to be addressed in two parts, one is when the data is for other than Indian visibility and the data within Indian visibility cone. When the area within Indian visibility cone is asked for we need to consider that a country like India which is very much in the use of Remote Sensing data needs data for its National projects like Crop estimation, Irrigation related, waste 
land mapping etc. These need data without gap and for the full country. Whenever the data for disasters is planned it is always at the cost of the other ongoing projects. Specially in case of RISAT-1 the ongoing projects look for specific angles, polarizations and look direction like FASAL requires data in Descending left look with 36 deg angle and dual polarization. Figure-4 shows the data requirement of FASAL.

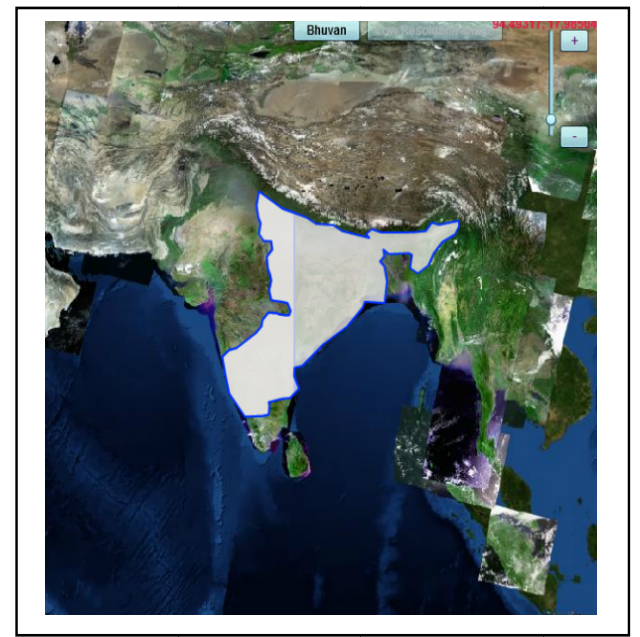

Figure-4: FASAL requirements with RISAT-1

The data other than the Indian visibility cone are always planned with the onboard tape recorder and the Antractica station ( Bharati) is used for down linking the data. The Indian station at Antarctica (Bharati), Larsemann Hills, $\left(69^{\circ} \mathrm{S}\right.$ and $76^{\circ} \mathrm{E}$ approx) has been used to establish a ground station to acquire Indian Remote Sensing satellites data and to communicate with to them. The station was successfully established in Dec 2012 with S/X-Band and established a communication link between Antarctica and two stations in India (NRSC, Shadnagar and NCAOR, Goa). The link supports transfer of satellite data @2x40 Mbps in near real time to NRSC and 2 way communication services@ @ Mbps to NRSC, Shadnagar and NCAOR, Goa simultaneously.

Antarctica station facilitates local coverage collections over the region for the benefit of scientists studying the Antarctica region. Due its location the IRS satellites are visible to the station for about 10 to 11 orbits therefore it provides additional orbits for data down linking.

Within $1 \mathrm{~h}$ of downlink the data is transferred from Bharati station to Shadnagar station. The data is processed and supplied to the user. The best time lies achieved is $4 \mathrm{~h}$ turnaround time for Philippines earthquake. The location of Antarctica station is shown in Figure-5.During 2012-13, 25 International Charter requests were serviced.

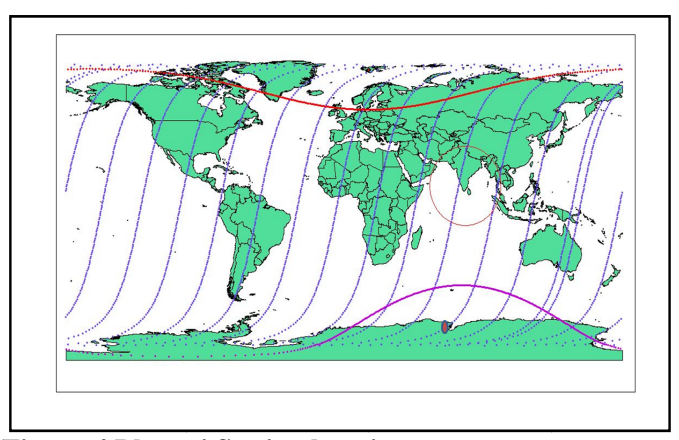

Figure-4 Bharati Station location.

\subsection{RISAT-1 programming}

RISAT-1 programming is more complex, given the variety of possible modes(resolution/swath) and incidences. Such variety provides a shorter access time (of the order of 2-3days), unless a specific constraint of angles are given. For example if the incidence angles of archived data are to be matched with future collection , this makes the planning complex and in turn reduces the collection capabilities.

For example for water-related applications, high incidence shall be selected for flood detection (to minimise water clutter, in the attempt to maximize the contrast between water and land) where as low incidence shall be selected for oil spills (to maximise sea clutter and therefore the contrast between polluted and clean water surfaces).

A trade-off between spatial resolution and swath has to be done, choosing the highest resolution while covering the area to be observed. Coupled with this the process becomes more complex when emergencies are to be combined with ongoing requests.

Based on the cloud movement and the estimated rain fall and occurrence for some of the events the satellites are requested to be tasked with an advance notice of few hours. In the years 2012-13, twelve states were either effected by floods or by Cyclone. With RISAT-1 all these were supported. This eventually disturbed the requirement for the other projects. Figure 5- shows the areas planned for floods in 2012-2013

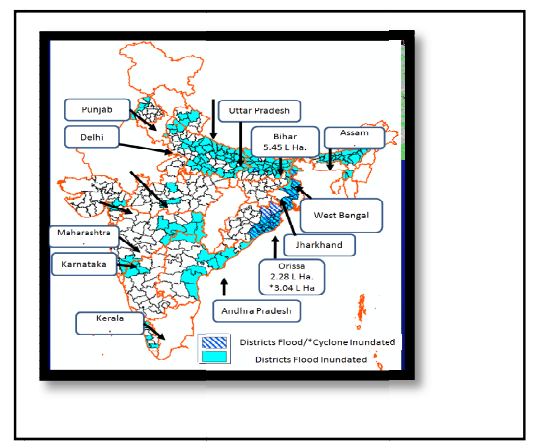

Figure :-5 RISAT-1 planned for disaster during 2012-13

When selecting radar acquisitions for the purpose of damage assessment, the matching of mode, node and polarization are very important (same incidence and resolution) between archives and new acquisitions as the variation information is derived from the comparison between acquisitions obtained before and after an event.

\section{Examples of disaster monitoring and risk management}

2.1 An example of flood monitoring using IRS constellation of satellites over Assam area.During third week of August, 2014, several areas in Assam state were inundated due to floods in Brahmaputra, Beki and other rivers. Kaziranga National Park also inundated by the flood waters of Brahmaputra River. The request for data collection was triggered on $6^{\text {th }}$ Sep 2014.

ISRO / NRSC programmed RISAT-1 satellite data of the area on $7^{\text {th }} \mathrm{Sep}, 9^{\text {th }} \mathrm{Sep}, 10^{\text {th }} \mathrm{Sep}, 11^{\text {th }}$ Sep with coarse resolution $50 \mathrm{~m}$ resolution. Pre event data was available on 14th August 2014. The flood inundation information was disseminated to the concerned Central and Assam State Govt. departments. The following satellite images show pre \& post flood situation in Nowgong, Sonitpur and other districts and the floods in Kaziranga National Park (Figure 6) 


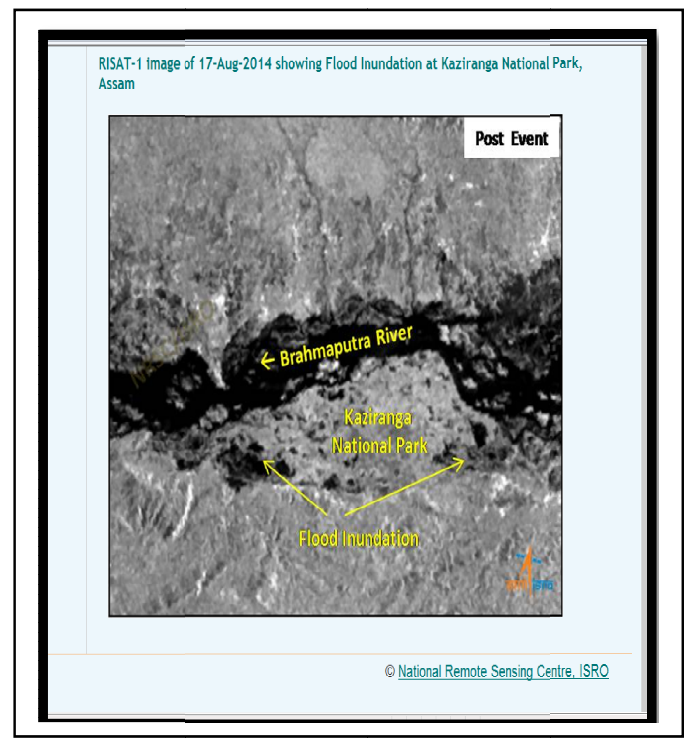

Figure-6 RISAT-1 - 17 Aug 2014 - Assam flood inundation

2.2 Jammu \& Kashmir floods: Severe floods were reported in Jammu \& Kashmir during first week of September, 2014. The request for emergency planning with all satellites was triggered on $6^{\text {th }}$ Sep $2014 \quad 6$ am IST. The earliest satellite that could track the area was Resourcesat- 1 AWiFS sensor. The satellite passed the area at 9.45 am on $6^{\text {th }}$ Sep 2014 and the product was supplied immediately. Heavy rains coupled with rise in the water levels of Jhelum river had led to flooding in the districts of Anantnag, Pulwama, Badgam, Kulgam, Rajouri etc. Heavy rain falls cause widespread flooding in the Kashmir valley. Therefore this required continuous monitoring of Jammu \& Kashmir area.RISAT-1 was planned for this area for all possible opportunities. The data was collected on $8^{\text {th }}$ Sep, $9^{\text {th }}$ Sep, $10^{\text {th }}$ Sep, $12^{\text {th }}$ Sep. $17^{\text {th }}$ Sep, $20^{\text {th }}$ Sep , $21^{\text {st }}$ Sep, 22 Sep \& $28^{\text {th }}$ Sep 2014.Optical satellites were also tasked, Cartosat-1 $10^{\text {th }} \mathrm{Sep}$, Cartosat-2 $10^{\text {th }} \mathrm{Sep}$, Resourcesat-2 $9^{\text {th }} \mathrm{Sep}, 14^{\text {th }} \mathrm{Sep}$ and $19^{\text {th }}$ Sep 2014.The requirement of continuous monitoring could be fulfilled with the constellation of IRS satellites but at the cost of disturbing the collections of other National projects. Figure 7 shows the J\&K flood inundation.

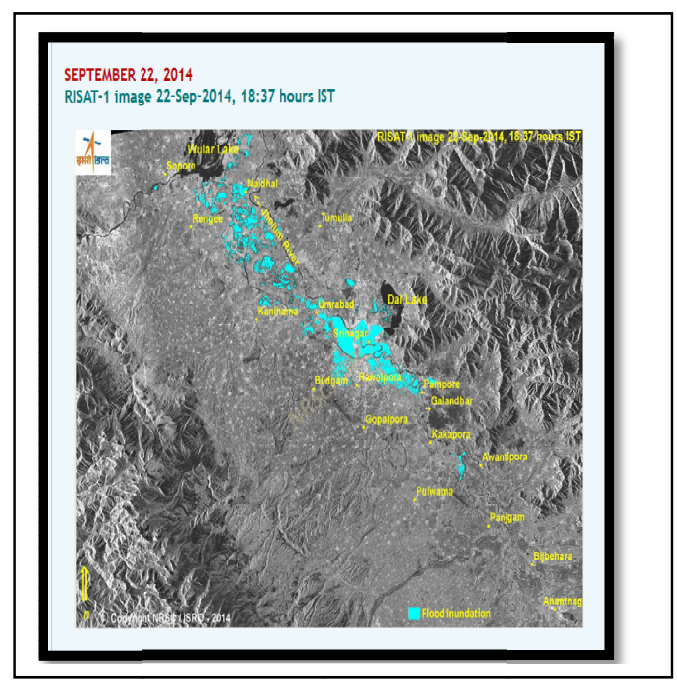

\subsection{HUDHUD cyclone:}

Cyclones are wind-systems of relatively low pressure which spiral inwards towards a centre in the lowest atmospheric levels and cause immense destruction and loss of life when they strike coastal areas. The Indian Metrological Department(IMD) gives a prediction of these cyclones. Based on the predictions the data requirement is initiated. Very Severe Cyclonic Storm 'HUDHUD' over west central Bay of Bengal made landfall at Andhra Pradesh coast on 12th October 2014.

The alerts of this were released by IMD on $6^{\text {th }}$ Oct 2014.The planning for the data collections were initiated on $6^{\text {th }}$ and continuously data was planned from $8^{\text {th }}$ Oct 2014 till $26^{\text {th }}$ Oct 2014.To have a complete assessment and a base map generated Coarse resolution followed by Medium resolution then high resolution data were planned. Figure 8 shows the planning of the satellite based on the track.

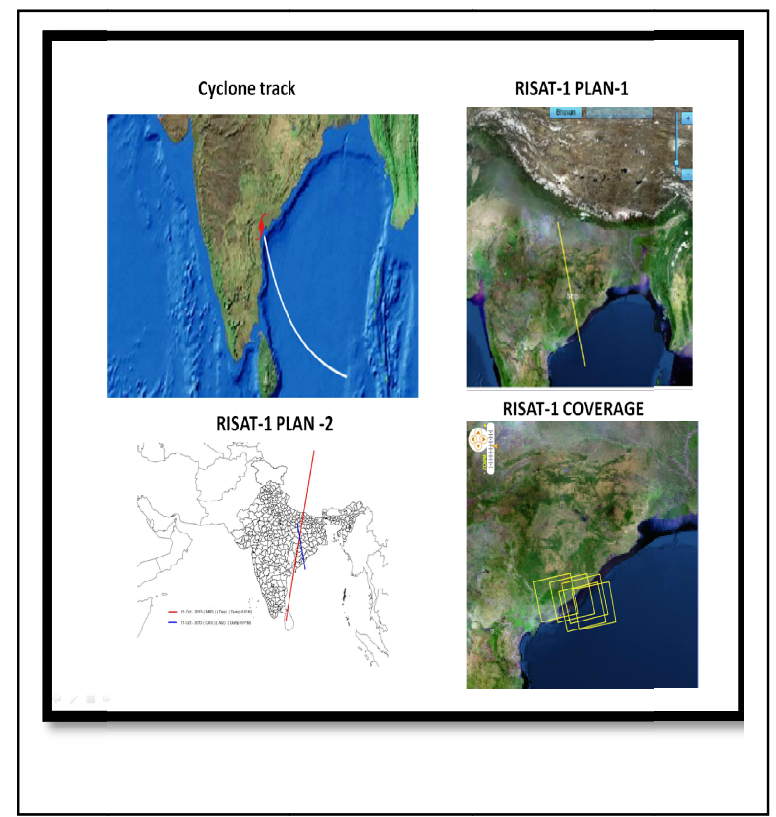

Figure-8 RISAT-1 - HUDHUD cyclone planning

\subsection{Floods in Nepal}

Heavy rain caused massive landslides from the hillside in Jure, Mankhaa of Sindhupal chowk district in Nepal's Central Region on 2 Aug 2014. The landslide created a high artificial dam across the Saptakoshi River, one of the main tributaries of the Koshi River, blocking the flow of water completely. With a large volume of water collecting above the dam, the risk of flash floods, which could also impact India's Bihar was a point of concern. Emergency was triggered on 3Aug 2014 for data collection and monitoring the area. Support was provided as shown in the table-2.Continuous monitoring was done and the maps were generated given till $19^{\text {th }}$ Aug 2014.

Figure-7 RISAT-1 - 22 Sep 2014 - Jammu \& Kashmir flood inundation 


\begin{tabular}{|c|c|c|c|}
\hline S.No & $\begin{array}{c}\text { DATE } \\
\text { PROGRAM M ED }\end{array}$ & \multicolumn{2}{|c|}{ Satellites } \\
\hline 1 & 4-Aug-14 & RS-2 & RISAT-II \\
\hline 2 & 5-Aug-14 & RS-2 & RISAT-II \\
\hline 3 & 6-Aug-14 & CARTOSAT-1 & \\
\hline 4 & 7-Aug-14 & & RISAT-II \\
\hline 5 & 8-Aug-14 & & RISAT-II \\
\hline 6 & 9-Aug-14 & RS-2 & RISAT-II \\
\hline 7 & 10-Aug-14 & CARTOSAT-2 & RISAT-1 \\
\hline 8 & 11-Aug-14 & & RISAT-II \\
\hline 9 & 12-Aug-14 & CARTOSAT-1 & RISAT-II \\
\hline 10 & 14-Aug-14 & CARTOSAT-2 & RS-2 \\
\hline 11 & 15-Aug-14 & & RISAT-1 \\
\hline 12 & 17-Aug-14 & CARTOSAT-1 & \\
\hline 13 & 18-Aug-14 & & RISAT-II \\
\hline 14 & 19-Aug-14 & CARTOSAT-2 & $\mathrm{RS}-2$ \\
\hline
\end{tabular}

\section{References:}

[1].Risk management using RS data: moving form scientific to operational applications by F . Sart etal

[2].NRSC web site www.nrsc.gov.in

[3.]K.Feigl, F.Sarti et al. « Estimating slip distribution forthe Izmit mainshock from coseismic GPS, SPOT and ERS-1 measurement ", submitted 07/09/2000 for publication on Bulletin of the Seismological Society of America, special issue on Izmit and Duzce Turkey Earthquakes

Table 2- IRS-Support for the Nepal floods

\section{Future Spatial Systems:}

ISRO is planning to launch both Microwave and Optical sensors , of medium and high resolution. Backup missions for Resoiurcesat-2 and RISAT-1 namely Resourcesat- $2 \mathrm{R}$ and RISAT-1A are planned. Apart from these series of high resolution missions are aimed at like Cartosat $3 / 3 \mathrm{~A} / 3 \mathrm{~B}$ with $0.25 \mathrm{~m}$ PAN and $1 \mathrm{~m}$ MX. Cartosat $1 \mathrm{~A} / 1 \mathrm{~B}$ with $1.25 \mathrm{~m}$ PAN stereo and 2.5m MX, Resourcesat-3 series with 20m MX .All these are highly flexible and together can give data with a gap of few hours provided they are phased.

\section{Conclusions:}

Remote sensing has become a tool for assessment or risk management and the demand for the same is increasing.2012-13 financial year witnessed flooding in 12 states of India. The IRS satellite constellation including the Microwave satellite gives opportunity of imaging any area on the globe almost every day. The optical sensors have the difficulty of the effect of the meteorological uncertainties. This demands continuous support of planning form the SAR sensors. Therefore RISAT-1 is mostly occupied with emergency planning. The existing constellation of satellites do not allow for a real time monitoring with few hours gap. Another limitation is non availability of simultaneous collections in optical and SAR data The situation will improve with the launch of dedicated constellation of satellites only for disaster monitoring and risk management.

\section{Acknowledgements}

We would like to thank Director NRSC Dr V K Dadhwal and Deputy Director MS B Lakshmi for allowing us to do this study. We are also thankful to Head Disaster Management Group, Mr G Srinivas Rao, NRSC for their inputs and support. 